\title{
Hidden chaotic attractors in a class of two-dimensional maps*
}

\author{
Haibo Jiang ${ }^{\mathrm{a} \dagger}$, Yang $\mathrm{Liu}^{\mathrm{b}}$, Zhouchao $\mathrm{Wei}^{\mathrm{c}}$, Liping Zhang ${ }^{\mathrm{a}}$ \\ a School of Mathematics and Statistics, Yancheng Teachers University, Yancheng 224002, China \\ bSchool of Engineering, Robert Gordon University, Garthdee Road, Aberdeen AB10 7GJ, UK \\ ${ }^{\mathrm{c}}$ School of Mathematics and Physics, China University of Geosciences, Wuhan 430074, China
}

\begin{abstract}
This paper studies the hidden dynamics of a class of two-dimensional maps inspired by the Hénon map. A special consideration is made to the existence of fixed points and their stabilities in these maps. Our concern focuses on three typical scenarios which may generate hidden dynamics, i.e. no fixed point, single fixed point, and two fixed points. A computer search program is employed to explore the strange hidden attractors in the map. Our findings show that the basins of some hidden attractors are tiny, so the standard computational procedure for localization is unavailable. The schematic exploring method proposed in this paper could be generalized for investigating hidden dynamics of high-dimensional maps.
\end{abstract}

Keywords: hidden dynamics; two-dimensional maps; fixed point; stability; coexistence.

\section{Introduction}

This paper is devoted to the investigation of hidden dynamics of a class of two-dimensional maps inspired by the Hénon map [1]. From a computation point of view, Leonov et al. proposed a new classification of attractor in [2,3]. If the basin of attraction for an attractor does not intersect with small neighborhoods of equilibria, this attractor is called a hidden attractor.

*accepted by Nonlinear Dynamics, DOI: 10.1007/s11071-016-2857-3, 2016.5.

${ }^{\dagger}$ Corresponding author. Tel.:+86 515 88239915, E-mail address: yctcjhb@126.com (H. Jiang), y.liu8@rgu.ac.uk (Y. Liu), weizhouchao@163.com (Z. Wei), yctczlp@126.com (L. Zhang). 
Otherwise, it is called a self-excited attractor. Self-excited attractors can be localized numerically by the standard computational procedure, i.e. choose a point from the unstable manifold in a neighborhood of an unstable equilibrium, and then trace the state of the attractor. While there is no regular way to predict the existence or coexistence of hidden attractors in a system since their basins of attraction are not connected with unstable equilibria. So one cannot guarantee the localization of a hidden attractor by following its trajectories with random initial conditions as its basin of attraction could be very small. Algorithms for finding hidden attractors in nonlinear systems have been proposed by Bragin et al. in [4], and one of these algorithms has been used to localize hidden attractors in the Chua's system in $[5,6]$.

In the last few years, there was a growing interest in studying hidden chaotic attractors in continuous systems. For example, Wei studied a simple three-dimensional autonomous chaotic system with no equilibria in [7]. The particularity of this system is that there exists a constant controller which can adjust the type of chaotic attractors. Jafari et al. [8] performed a systematic search to find additional three-dimensional chaotic systems with quadratic nonlinearties and no equilibria. Wei et al. studied a new four-dimensional hyperchaotic system by extending the generalized diffusionless Lorenz equations in [9], and the new model did not show any equilibria but two-scroll hyperchaos with chaotic, quasiperiodic and periodic dynamics. In [10], Molaie et al. found that a stable equilibrium point coexisted with 23 simple chaotic flows with quadratic nonlinearities by using the Routh-Hurwitz stability criterion and a systematic computer search. Wei and Zhang [11] reported the finding of a four-dimensional non-Sil'nikov autonomous system with three quadratic nonlinearities, and observed hidden hyperchaotic attractors with one stable equilibrium. In [12], Wei and Yang studied a new three-dimensional autonomous chaotic system which displayed double-scroll chaotic attractors in a very wide parameter domain with two stable equilibria. Moreover, a line equilibrium has been found in the nine simple chaotic flows with quadratic nonlinearities in [13], and Wang and Chen proposed a method in [14] for constructing the chaotic system with pre-assigned number of equilibria. In the meantime, the coexistence of hidden attractors has attracted great attention by many researchers, e.g. [15-19]. In [15, 16], the rare and hidden attractors in the externally excited van der Pol-Duffing oscillator have been investigated by using the concept of perpetual points in [17]. In [18], a coexisting stable limit cycle was found in a chaotic system which has only one stable equilibrium. Li and Sprott [19] studied a new four-dimensional simplified Lorenz system and obtained that it had an attracting torus in some regions of parameter space coexisting with either a symmetric pair of strange 
attractors or with a symmetric pair of limit cycles whose basin boundaries had an intricate fractal structure. In [20], hidden attractors which coexisted with a stable equilibrium were observed in a drilling system indicating that such hidden oscillations may cause costly drilling failure. In addition, hidden attractors in multi-stroll chaotic systems [21-23] have also been studied in [24].

On the other hand, dynamics of discrete-time maps, such as the Logistic map and the Hénon map, have been studied extensively in different disciplines fueled by their broad applications in economics, biology, and engineering (see [25-28] for examples). However, there are very few results on hidden attractors in discrete-time maps. In [29], three second-order counterexamples to the discrete-time Kalman conjecture were constructed and hidden stable periodic solutions were shown for these examples. Zhusubaliyev et al. [30] studied the multistability and hidden attractors in a multilevel DC/DC converter which was reduced to the analysis of the twodimensional piecewise-smooth map with multiple borders by integrating the equations of motion for the continuous-time system from switching event to switching event. In [31], some hidden attractors in one-dimensional map has been introduced by extending the Logistic map. In this paper, we will study a class of two-dimensional maps and explore their hidden attractors. Our main purpose is to devise a schematic approach for investigating hidden attractors in discretetime systems. The findings would allow one to study the mechanisms of hidden dynamics and the evolution of their basins of attraction in high-dimensional discrete-time systems.

The rest of this paper is organized as follows. In Section 2, the mathematical model of a class of two-dimensional maps is given, and the existence and the stability of its fixed points are studied. The strange hidden attractors with no fixed point, and with a single stable fixed point are investigated in Section 3 and 4, respectively. Finally, some conclusions are drawn in Section 5 .

\section{System model and fixed points}

\subsection{System model}

Inspired by the Hénon map, we consider a class of two-dimensional map which is described by the following difference equation

$$
\left\{\begin{array}{l}
x_{k+1}=y_{k}, \\
y_{k+1}=a_{1} x_{k}+a_{2} y_{k}+a_{3} x_{k}^{2}+a_{4} y_{k}^{2}+a_{5} x_{k} y_{k}+a_{6},
\end{array}\right.
$$


where $a_{1}, a_{2}, a_{3}, a_{4}, a_{5}, a_{6}$ are real coefficients.

The Jacobian matrix of the map is given as

$$
J=\left[\begin{array}{ll}
0 & 1 \\
a_{1}+2 a_{3} x_{k}+a_{5} y_{k} & a_{2}+2 a_{4} y_{k}+a_{5} x_{k}
\end{array}\right]
$$

and the characteristic equation of the Jacobian matrix can be calculated as

$$
\operatorname{det}(\lambda I-J)=\lambda^{2}-\operatorname{tr}(J) \lambda+\operatorname{det}(J)=0,
$$

where $\operatorname{det}(J)=-\left(a_{1}+2 a_{3} x_{k}+a_{5} y_{k}\right)$ is the determinant of the Jacobian matrix, and $\operatorname{tr}(J)=$ $a_{2}+2 a_{4} y_{k}+a_{5} x_{k}$ is the trace of the Jacobian matrix. According to the theory of matrix, the sum of the eigenvalues of Jacobian matrix is equal to $\operatorname{tr}(J)$ and the product of the eigenvalues of Jacobian matrix is equal to $\operatorname{det}(J)$.

\subsection{Fixed points and stability analysis}

The fixed point of the map $(x, y)$ must satisfy the following conditions

$$
\left\{\begin{array}{l}
x=y, \\
y=a_{1} x+a_{2} y+a_{3} x^{2}+a_{4} y^{2}+a_{5} x y+a_{6} .
\end{array}\right.
$$

Then the problem of finding fixed point can be transformed into solving the following equation with respect to $y$

$$
\left(a_{3}+a_{4}+a_{5}\right) y^{2}+\left(a_{1}+a_{2}-1\right) y+a_{6}=0 .
$$

Assume that there exists a fixed point $(x, y)$ of the map (1). The fixed point is stable if the roots $\lambda_{1}, \lambda_{2}$ of the characteristic equation satisfy that $\left|\lambda_{1,2}\right|<1$. To establish the stability conditions of the fixed points, the following lemma is used.

Lemma 1. [26-28] The fixed point $(x, y)$ of the map (1) is stable if the following conditions are satisfied

$$
\left\{\begin{array}{l}
\operatorname{det}(J)-1<0, \\
\operatorname{tr}(J)-\operatorname{det}(J)-1<0, \\
\operatorname{tr}(J)+\operatorname{det}(J)+1>0 .
\end{array}\right.
$$

where $\operatorname{det}(J)=-\left(a_{1}+2 a_{3} x+a_{5} y\right)$ is the determinant of the Jacobian matrix, and $\operatorname{tr}(J)=$ $a_{2}+2 a_{4} y+a_{5} x$ is the trace of the Jacobian matrix. 
Remark 1. If $\operatorname{det}(J)=1, \lambda_{1} \lambda_{2}=1$. If $\operatorname{tr}(J)+\operatorname{det}(J)+1=0$, there is a real root $\lambda=-1$. If $\operatorname{tr}(J)-\operatorname{det}(J)-1=0$, there is a real $\operatorname{root} \lambda=-1$.

If $a_{3}+a_{4}+a_{5} \neq 0, \Delta=\left(a_{1}+a_{2}-1\right)^{2}-4\left(a_{3}+a_{4}+a_{5}\right) a_{6}$ is denoted as the discriminant of Eq. (5).

\subsubsection{Case 1: no fixed point}

(1) No fixed point I (NF I)

If $a_{3}+a_{4}+a_{5}=0, a_{1}+a_{2}-1=0$ and $a_{6} \neq 0$, Eq. (5) has no solution, and the map (1) has no fixed point. For any existence of attractors, they must be hidden as the basins of attraction of these attractors do not contain any fixed point.

(2) No fixed point II (NF II)

If $a_{3}+a_{4}+a_{5} \neq 0$ and $\Delta<0$, Eq. (5) has no solution, and the map (1) has no fixed point. Again, if there exists an attractor, it must be hidden since the basin of attraction of this attractor does not contain any fixed point.

\subsubsection{Case 2: single fixed point}

(1) Single fixed point I (SF I)

If $a_{3}+a_{4}+a_{5}=0$ and $a_{1}+a_{2}-1 \neq 0$, Eq. (5) has a single solution $y=-\frac{a_{6}}{a_{1}+a_{2}-1}$, and the map (1) has a fixed point $\left(x^{*}, y^{*}\right)$, where $x^{*}=y^{*}=-\frac{a_{6}}{a_{1}+a_{2}-1}$. This fixed point is stable if the eigenvalues $\lambda_{1}, \lambda_{2}$ of the Jacobian matrix $J_{1}=\left.J\right|_{x=x^{*}, y=y^{*}}$ lie in the unit circle, i.e. $\left|\lambda_{i}\right|<1$, where $i=1,2$. By Lemma 1 , the fixed point $\left(x^{*}, y^{*}\right)$ is stable if the following conditions are satisfied

$$
\left\{\begin{array}{l}
\operatorname{det}\left(J_{1}\right)-1<0, \\
\operatorname{tr}\left(J_{1}\right)-\operatorname{det}\left(J_{1}\right)-1<0, \\
\operatorname{tr}\left(J_{1}\right)+\operatorname{det}\left(J_{1}\right)+1>0,
\end{array}\right.
$$

which leads to

$$
\left\{\begin{array}{l}
\left(a_{1}-a_{1} a_{2}+2 a_{3} a_{6}+a_{5} a_{6}-a_{1}^{2}\right) /\left(a_{1}+a_{2}-1\right)-1<0, \\
a_{2}-\left(a_{1}-a_{1} a_{2}+2 a_{3} a_{6}+a_{5} a_{6}-a_{1}^{2}\right) /\left(a_{1}+a_{2}-1\right) \\
\quad-\left(2 a_{4} a_{6}\right) /\left(a_{1}+a_{2}-1\right)-\left(a_{5} a_{6}\right) /\left(a_{1}+a_{2}-1\right)-1<0, \\
a_{2}+\left(a_{1}-a_{1} a_{2}+2 a_{3} a_{6}+a_{5} a_{6}-a_{1}^{2}\right) /\left(a_{1}+a_{2}-1\right) \\
\quad-\left(2 a_{4} a_{6}\right) /\left(a_{1}+a_{2}-1\right)-\left(a_{5} a_{6}\right) /\left(a_{1}+a_{2}-1\right)+1>0 .
\end{array}\right.
$$


Suppose that there exist some other attractors except this single fixed point. Since the fixed point $\left(x^{*}, y^{*}\right)$ is stable and the basins of attraction cannot contain any fixed point, these attractors of the map except this single fixed point are hidden.

(2) Single fixed point II (SF II)

If $a_{3}+a_{4}+a_{5} \neq 0$ and $\Delta=0$, Eq. (5) has two equal real roots $y_{1}=y_{2}=-\frac{a_{1}+a_{2}-1}{2\left(a_{3}+a_{4}+a_{5}\right)}$, and the map (1) has a fixed point $\left(x^{*}, y^{*}\right)$, where $x^{*}=y^{*}=-\frac{a_{1}+a_{2}-1}{2\left(a_{3}+a_{4}+a_{5}\right)}$. However, the Jacobian

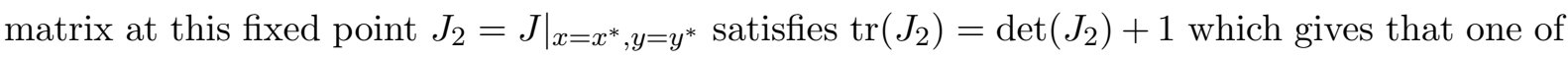
the eigenvalues of the Jacobian matrix equals to one. Thus the fixed point $\left(x^{*}, y^{*}\right)$ is not stable.

\subsubsection{Case 3: Two fixed points (TF)}

If $a_{3}+a_{4}+a_{5} \neq 0$ and $\Delta>0$, Eq. (5) has two distinct real roots $y_{1,2}=\frac{-\left(a_{1}+a_{2}-1\right) \pm \sqrt{\Delta}}{2\left(a_{3}+a_{4}+a_{5}\right)}$, and the map (1) has two fixed points $\left(x_{1,2}^{*}, y_{1,2}^{*}\right)$, where $x_{1,2}^{*}=y_{1,2}^{*}=\frac{-\left(a_{1}+a_{2}-1\right) \pm \sqrt{\Delta}}{2\left(a_{3}+a_{4}+a_{5}\right)}$. These two fixed points are stable if the eigenvalues $\lambda_{1}, \lambda_{2}$ of the Jacobian matrices $J_{3}=\left.J\right|_{x=x_{1}^{*}, y=y_{1}^{*}}$ and $J_{4}=\left.J\right|_{x=x_{2}^{*}, y=y_{2}^{*}}$ all lie in the unit circle, i.e. $\left|\lambda_{i}\right|<1$, where $i=1,2$. Thus both fixed points are stable if the following conditions are satisfied

$$
\left\{\begin{array}{l}
\operatorname{det}\left(J_{3}\right)-1<0, \\
\operatorname{tr}\left(J_{3}\right)-\operatorname{det}\left(J_{3}\right)-1<0 \\
\operatorname{tr}\left(J_{3}\right)+\operatorname{det}\left(J_{3}\right)+1>0 \\
\operatorname{det}\left(J_{4}\right)-1<0 \\
\operatorname{tr}\left(J_{4}\right)-\operatorname{det}\left(J_{4}\right)-1<0 \\
\operatorname{tr}\left(J_{4}\right)+\operatorname{det}\left(J_{4}\right)+1>0
\end{array}\right.
$$

However, by using the command "simplify" in the scientific computing software MATLAB, the conditions are "FALSE", which implies that there is a contraction in these inequalities. So it indicates that the map cannot have two stable fixed points.

\section{Strange attractors with no fixed point}

A computer search program [32] was used to explore the strange attractors with no fixed point. In this section, we will show some typical examples, and the elemental dynamics of the map will be studied. The Lyapunov exponents of the chaotic attractors were computed by using the same method given in [33-36]. If the Lyapunov exponents of the point $p_{0}=\left(x_{0}, y_{0}\right)$ on the chaotic attractors are $L_{1}\left(p_{0}\right)$ and $L_{2}\left(p_{0}\right)$, i.e., $L_{1}\left(p_{0}\right)>0$ and $L_{2}\left(p_{0}\right)<0$, the local Lyapunov 
(Kaplan-Yorke) dimension $\operatorname{dim}_{L} p_{0}$ can be given as $\operatorname{dim}_{L} p_{0}=1-L_{1}\left(p_{0}\right) / L_{2}\left(p_{0}\right)$. In this paper, a grid of points on chaotic attractors were used to find the maximum of the local Lyapunov dimensions, i.e., $\overline{\operatorname{dim}}_{L}=\max _{p_{0} \in B}\left(\operatorname{dim}_{L} p_{0}\right)$, where $B$ is the set of points on chaotic attractors with a grid step $h=0.1$ of the phase space. In the reorthogonalization procedure, the time-step and the number of iterations were chosen as 10 and 100000, respectively. For the details of the computing procedure, readers could refer to [33-36].

\subsection{NF I}

Nine typical examples for the case with NF I are presented in Table 1 in which the initial value $\left(x_{0}, y_{0}\right)$, the Lyapunov exponents (Les) and the maximum of the local Lyapunov dimensions $\left(\overline{\operatorname{dim}}_{L}\right)$ are given. It can be verified that all the maps listed in Table 1 satisfy $a_{3}+a_{4}+a_{5}=0$, $a_{1}+a_{2}-1=0$ and $a_{6} \neq 0$ indicating that they have no fixed point. Thus the attractors obtained in the map are hidden. As shown in $[37,38]$, positive Lyapunov exponents may not lead to chaos since there are known examples with the so-called Perron effects of sign reversal for the largest Lyapunov exponent. However, because the considered map (1) belongs to a class of autonomous discrete systems with real coefficients, positive Lyapunov exponents are still adopted as an indicator of chaos in this paper. It can be seen from Table 1 that all the maximal Lyapunov exponents are positive, so the maps with the given initial values are all chaotic.

The basins of attraction for the examples of the map with NF I listed in Table 1 are presented in Fig. 1, where the chaotic attractors, the period-two orbits, and the period-ten orbits are marked by black, red, and blue dots, respectively, and the basins of unbound, the chaotic attractors, the period-two orbits, and the period-ten orbits are shown in cyan, white, yellow, and orange, respectively. As can be seen from Fig. 1(a)-(e), there is one chaotic attractor for each map and the basins of the chaotic attractors in Fig. 1(a)-(d) are large. However, the basin of the chaotic attractor in Fig. 1(e) is very small such that it is difficult to be obtained by using the standard computing method. In Fig. 1(f) and (g), a period-two attractor and a chaotic attractor coexist, and the basins of the chaotic attractors are very small. In Fig. 1(i), a period-two attractor, a period-ten attractor, and a chaotic attractor coexist, and the basin of the chaotic attractor is also very small. 
(a)

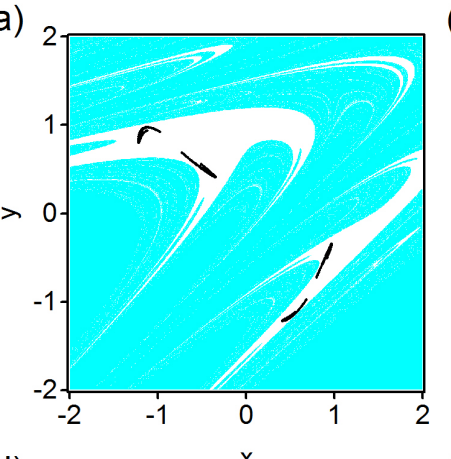

(d)

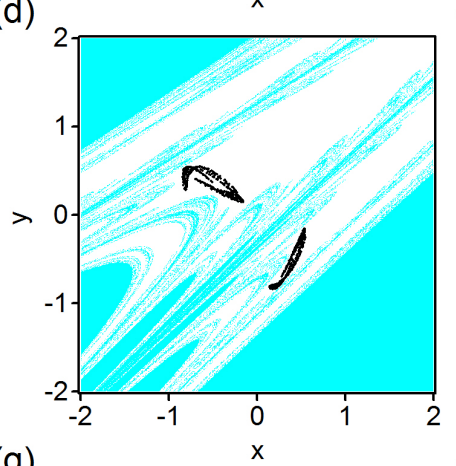

(g)

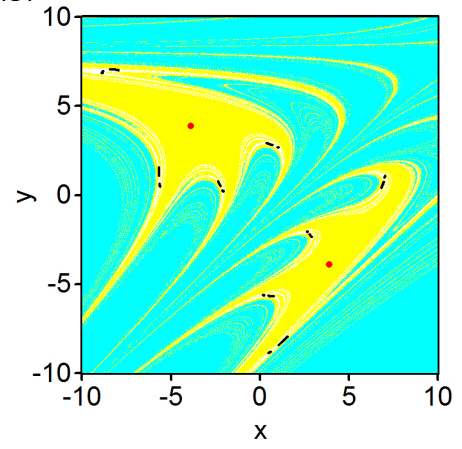

(b)

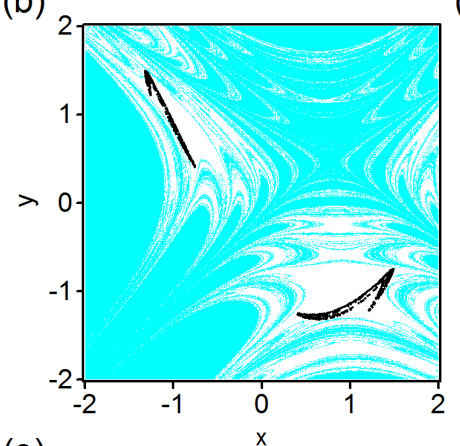

(e)

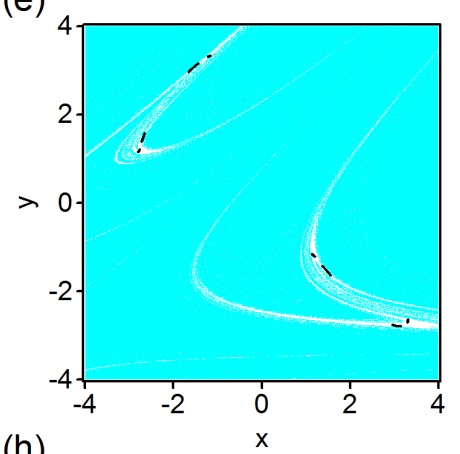

(h)

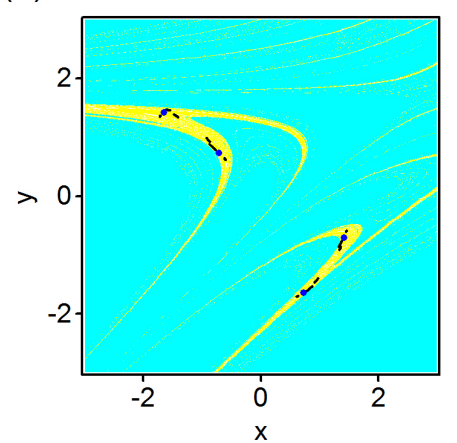

(c)

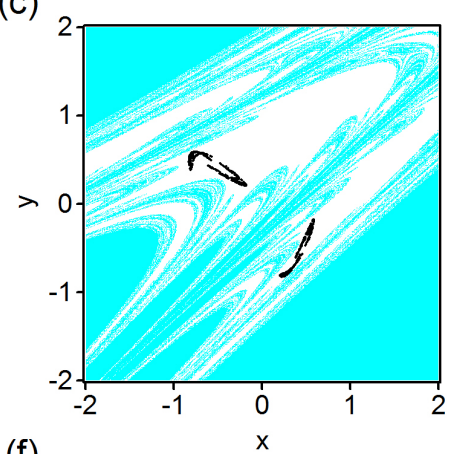

(f)
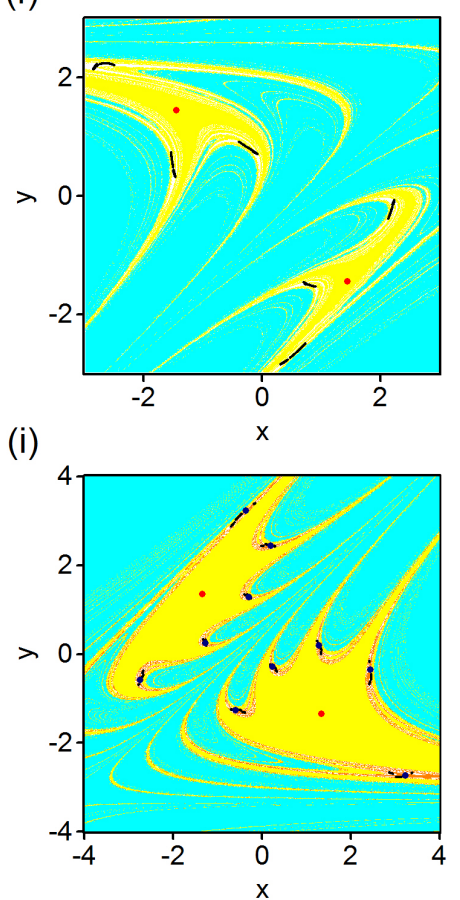

Fig. 1. Basins of attraction for the examples of the map with NF I listed in Table 1. The chaotic attractors, the period-two attractors, and the period-ten attractor are marked by black, red, and blue dots, respectively. The basins of unbound, the chaotic attractors, the period-two attractors, and the period-ten attractor are shown in cyan, white, yellow and orange, respectively. 
Table 1. Examples of the two-dimensional map with NF I

\begin{tabular}{|c|c|c|c|c|}
\hline Case & Maps & $\left(x_{0}, y_{0}\right)$ & Les & $\overline{\operatorname{dim}}_{L}$ \\
\hline $\mathrm{NFI}_{a}$ & $\begin{array}{l}x_{k+1}=y_{k} \\
y_{k+1}=x_{k}+0.2 x_{k}^{2}+0.71 y_{k}^{2}-0.91 x_{k} y_{k}-1.14\end{array}$ & $(0.93,-0.44)$ & $\begin{array}{c}0.0623 \\
-0.3248\end{array}$ & 1.1947 \\
\hline $\mathrm{NFI}_{b}$ & $\begin{array}{l}x_{k+1}=y_{k} \\
y_{k+1}=x_{k}-0.6 x_{k}^{2}+0.74 y_{k}^{2}-0.14 x_{k} y_{k}-0.33\end{array}$ & $(-0.78,0.45)$ & $\begin{array}{c}0.0827 \\
-0.2349\end{array}$ & 1.3572 \\
\hline $\mathrm{NFI}_{c}$ & $\begin{array}{l}x_{k+1}=y_{k} \\
y_{k+1}=x_{k}+0.51 x_{k}^{2}+y_{k}^{2}-1.51 x_{k} y_{k}-0.74\end{array}$ & $(-0.81,0.51)$ & $\begin{array}{c}0.0886 \\
-0.2448\end{array}$ & 1.3649 \\
\hline $\mathrm{NFI}_{d}$ & $\begin{array}{l}x_{k+1}=y_{k} \\
y_{k+1}=x_{k}+0.6 x_{k}^{2}+y_{k}^{2}-1.6 x_{k} y_{k}-0.72\end{array}$ & $(-0.26,0.18)$ & $\begin{array}{c}0.1012 \\
-0.2067\end{array}$ & 1.4932 \\
\hline $\mathrm{NFI}_{e}$ & $\begin{array}{l}x_{k+1}=y_{k} \\
y_{k+1}=x_{k}-0.3 y_{k}^{2}+0.3 x_{k} y_{k}+2.98\end{array}$ & $(3.02,-2.78)$ & $\begin{array}{c}0.0430 \\
-0.3523\end{array}$ & 1.1229 \\
\hline $\mathrm{NFI}_{f}$ & $\begin{array}{l}x_{k+1}=y_{k} \\
y_{k+1}=x_{k}+0.38 y_{k}^{2}-0.38 x_{k} y_{k}-1.6\end{array}$ & $(-0.07,0.71)$ & $\begin{array}{c}0.0535 \\
-0.2455\end{array}$ & 1.2188 \\
\hline $\mathrm{NFI}_{g}$ & $\begin{array}{l}x_{k+1}=y_{k} \\
y_{k+1}=x_{k}+0.12 y_{k}^{2}-0.12 x_{k} y_{k}-3.62\end{array}$ & $(1.03,2.68)$ & $\begin{array}{c}0.0303 \\
-0.1589\end{array}$ & 1.1928 \\
\hline $\mathrm{NFI}_{h}$ & $\begin{array}{l}x_{k+1}=y_{k} \\
y_{k+1}=x_{k}+0.57 y_{k}^{2}-0.57 x_{k} y_{k}-1.54\end{array}$ & $(-0.6,0.62)$ & $\begin{array}{c}0.0489 \\
-0.3468\end{array}$ & 1.1415 \\
\hline $\mathrm{NFI}_{i}$ & $\begin{array}{l}x_{k+1}=y_{k} \\
y_{k+1}=x_{k}-0.3 y_{k}^{2}+0.3 x_{k} y_{k}+1.09\end{array}$ & $(1.33,0.12)$ & $\begin{array}{c}0.0154 \\
-0.1079\end{array}$ & 1.1430 \\
\hline
\end{tabular}

\subsection{NF II}

Six typical examples for the map with NF II are presented in Table 2 where their initial values, the Lyapunov exponents (Les) and the maximum of the local Lyapunov dimensions $\left(\overline{\operatorname{dim}}_{L}\right)$ are given. It should be noted that all these maps satisfy $a_{3}+a_{4}+a_{5} \neq 0$ and $\Delta<0$ so that they do not have any fixed point and the attractors of these maps are hidden. The basins of attraction of these maps are shown in Fig. 2, where the chaotic attractors are marked by black dots, and the basins of unbound and the chaotic attractors are given in cyan and white, respectively. It is worth noting that the maximal Lyapunov exponents in Table 2 are positive, so the maps with the given initial values are all chaotic.

\section{Strange attractors with a single stable fixed point}

Four typical examples for this case are presented in Table 3 in which the fixed points, the absolute values of the eigenvalues of the Jacobian matrix at the fixed points, the initial values, 

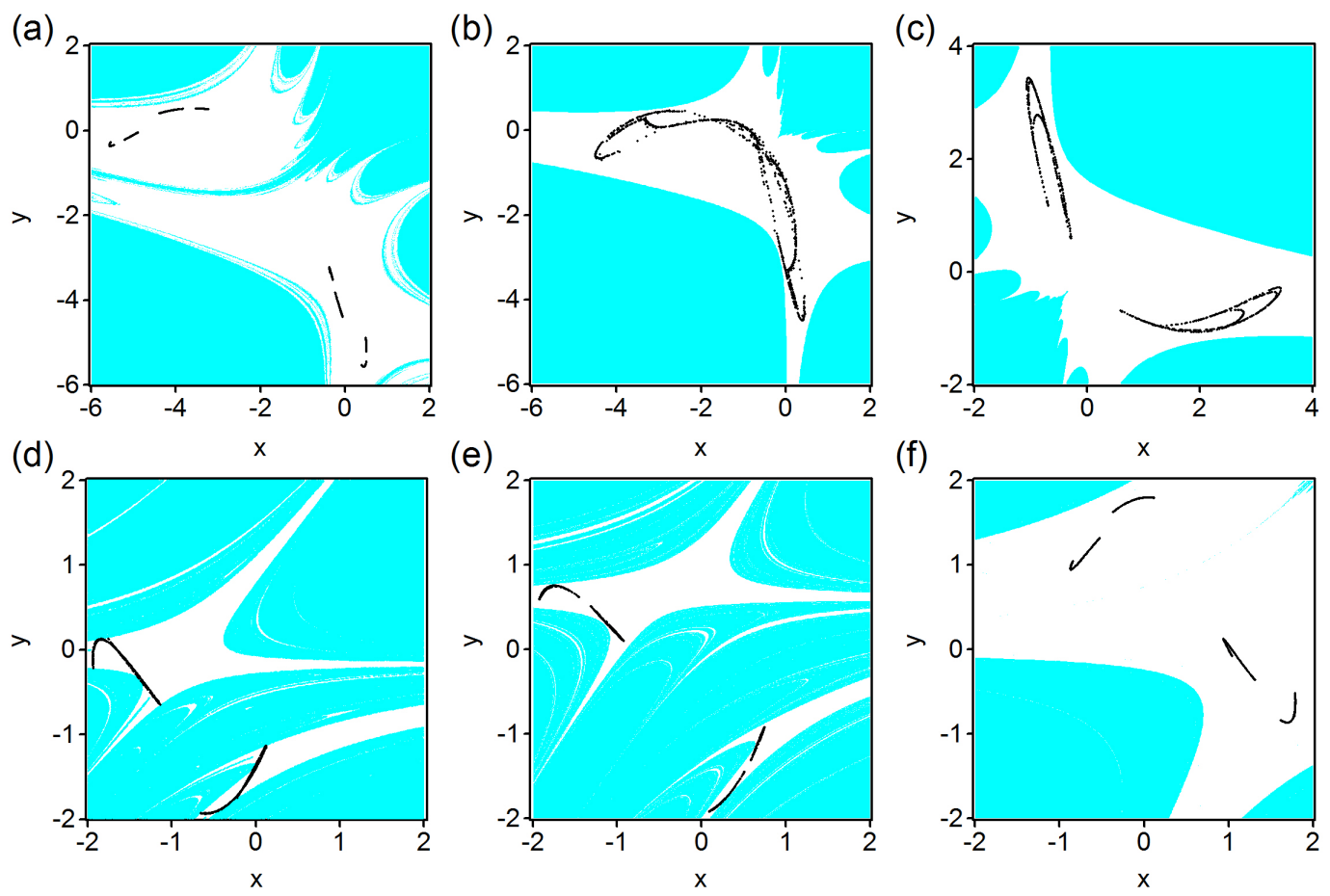

Fig. 2. Basins of attraction for the examples of the map with NF II listed in Table 2. The chaotic attractors are marked by black dots, and the basins of unbound and the chaotic attractors are shown in cyan and white, respectively. 
Table 2. Examples of the two-dimensional map with NF II

\begin{tabular}{|c|c|c|c|c|}
\hline Case & \multicolumn{1}{|c|}{ Maps } & $\left(x_{0}, y_{0}\right)$ & Les & $\overline{\operatorname{dim}}_{L}$ \\
\hline $\mathrm{NFII}_{a}$ & $\left\{\begin{array}{l}x_{k+1}=y_{k} \\
y_{k+1}=2.16 x_{k}+0.22 x_{k}^{2}-0.02 y_{k}^{2}+0.6 x_{k} y_{k}+0.76\end{array}\right.$ & $(0.51,-5.04)$ & 0.0467 & 1.0419 \\
\hline $\mathrm{NFII}_{b}$ & $\left\{\begin{array}{l}x_{k+1}=y_{k} \\
y_{k+1}=1.77 x_{k}-0.08 y_{k}+0.23 x_{k}^{2}+x_{k} y_{k}+0.1\end{array}\right.$ & $(-0.52,-1.17)$ & 0.1927 & 1.4525 \\
\hline $\mathrm{NFII}_{c}$ & $\left\{\begin{array}{l}x_{k+1}=y_{k} \\
y_{k+1}=0.9 x_{k}-0.72 y_{k}-0.3 x_{k}^{2}-x_{k} y_{k}-0.15\end{array}\right.$ & $(2.78,-0.75)$ & 0.1805 & 1.2252 \\
\hline $\mathrm{NFII}_{d}$ & $\left\{\begin{array}{l}x_{k+1}=y_{k} \\
y_{k+1}=-0.16 x_{k}+0.7 y_{k}^{2}-x_{k} y_{k}-1.67\end{array}\right.$ & $(-0.07,-1.5)$ & 0.2069 & 1.2009 \\
\hline $\mathrm{NFII}_{e}$ & $\left\{\begin{array}{l}x_{k+1}=y_{k} \\
y_{k+1}=0.6 x_{k}+0.49 y_{k}^{2}-x_{k} y_{k}-1.46\end{array}\right.$ & $(1.78,-0.79)$ & -1.0328 & 0.1219 \\
\hline $\mathrm{NFII}_{f}$ & $\left\{\begin{array}{l}x_{k+1}=y_{k} \\
y_{k+1}=-0.73 y_{k}-0.37 y_{k}^{2}+0.81 x_{k} y_{k}+1.79\end{array}\right.$ & -0.7715 & 1.1589 \\
\hline
\end{tabular}

the Lyapunov exponents (Les) and the maximum of the local Lyapunov dimensions $\left(\overline{\operatorname{dim}}_{L}\right)$ are given. Since all the maps in Table 3 satisfy $a_{3}+a_{4}+a_{5}=0$ and $a_{1}+a_{2}-1 \neq 0$, they have a single fixed point. Moreover, all the absolute values of the eigenvalues of the Jacobian matrix at the fixed points are less than 1, so these fixed points are stable.

The basins of attraction for the maps in Table 3 are presented in Fig. 3, where the chaotic attractors and the fixed points are shown by black and red dots, and the basins of unbound, the chaotic attractors, and the fixed points are depicted in cyan, white, and yellow, respectively. It can be see from Table 3 that the maximal Lyapunov exponents are positive, all the attractors obtained by the given initial values are chaotic. As can be seen from the figure, the basins of attraction of the hidden chaotic attractors are relative smaller than the ones of the fixed points in Fig. 3(a)-(c), while the basin of the hidden chaotic attractor in Fig. 3(d) is large and the one for the fixed point is tiny.

\section{Conclusion}

The hidden dynamics of a class of two-dimensional maps was studied in this paper. The existence of fixed points and their stabilities of these two-dimensional maps were considered firstly. Then different types of fixed points related to possible hidden dynamics were considered in three cases, i.e., no fixed point (NF), single fixed point (SF) and two fixed points (TF). Finally, 

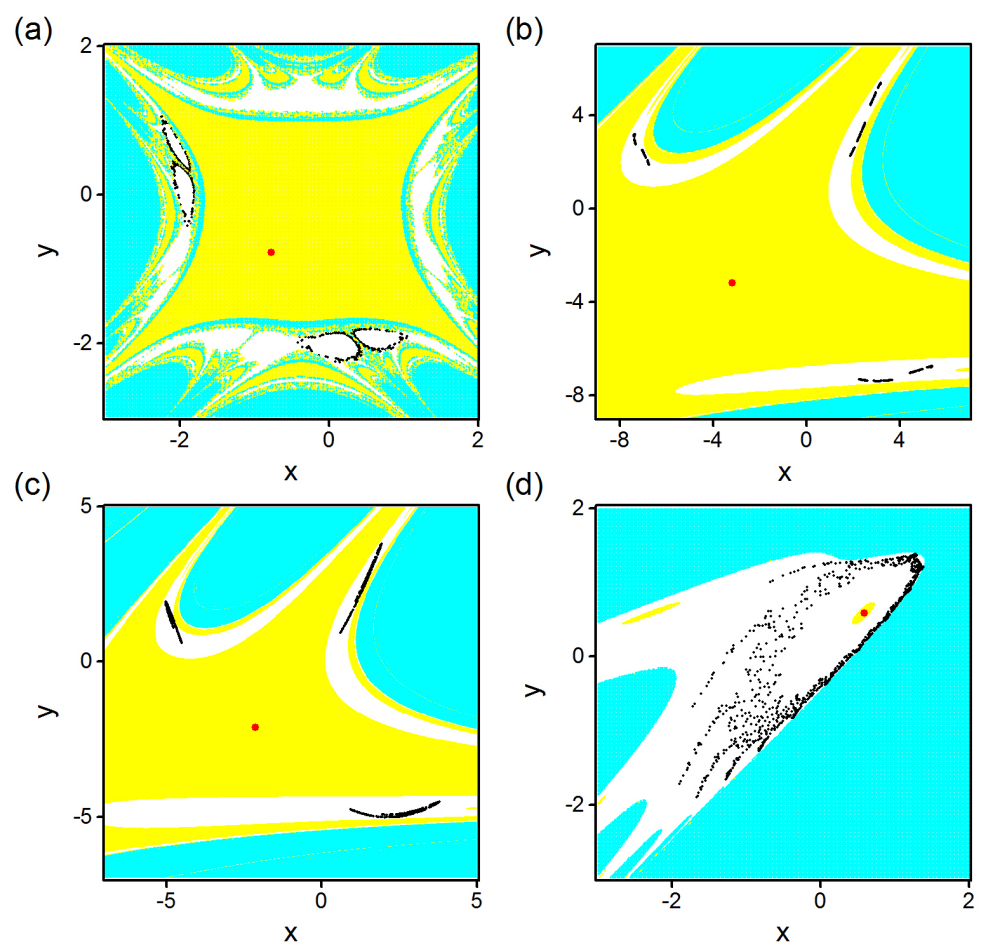

Fig. 3. Phase-basin portraits of the maps listed in the Table 3. The chaotic attractors and fixed points are denoted in black and red. The basin of unbound, chaotic attractors and fixed points are indicated in cyan, white and yellow, respectively. 
Table 3. Examples of the two-dimensional map with SF I

\begin{tabular}{|c|c|c|c|c|c|c|}
\hline Case & Maps & Fixed point & $\left|\lambda_{i}\right|$ & $\left(x_{0}, y_{0}\right)$ & Les & $\overline{\operatorname{dim}}_{L}$ \\
\hline $\mathrm{SFI}_{a}$ & $\begin{aligned} x_{k+1}= & y_{k} \\
y_{k+1}=- & 0.33 x_{k}+0.17 y_{k}-0.48 x_{k}^{2} \\
& +0.47 y_{k}^{2}+0.01 x_{k} y_{k}-0.9\end{aligned}$ & $\begin{array}{l}-0.7759 \\
-0.7759\end{array}$ & $\begin{array}{l}0.4146 \\
0.9817\end{array}$ & $\begin{array}{c}0.32 \\
-1.85\end{array}$ & $\begin{array}{c}0.0107 \\
-0.0279\end{array}$ & 1.3937 \\
\hline $\mathrm{SFI}_{b}$ & $\begin{aligned} x_{k+1}= & y_{k} \\
y_{k+1}= & -0.84 x_{k}+0.15 y_{k}^{2} \\
& \quad-0.15 x_{k} y_{k}-5.85\end{aligned}$ & $\begin{array}{l}-3.1793 \\
-3.1793\end{array}$ & $\begin{array}{l}0.6026 \\
0.6026\end{array}$ & $\begin{array}{c}4.61 \\
-6.99\end{array}$ & $\begin{array}{r}0.0389 \\
-0.3537\end{array}$ & 1.1112 \\
\hline $\mathrm{SFI}_{c}$ & $\begin{aligned} x_{k+1}= & y_{k} \\
y_{k+1}= & -0.99 x_{k}+0.23 y_{k}^{2} \\
& \quad-0.23 x_{k} y_{k}-4.24\end{aligned}$ & $\begin{array}{l}-2.1307 \\
-2.1307\end{array}$ & $\begin{array}{l}0.7071 \\
0.7071\end{array}$ & $\begin{array}{r}3.15 \\
-4.82\end{array}$ & $\begin{array}{r}0.1111 \\
-0.5854\end{array}$ & 1.1913 \\
\hline $\mathrm{SFI}_{d}$ & $\begin{aligned} x_{k+1}= & y_{k} \\
y_{k+1}=- & 1.29 x_{k}+2 y_{k}-0.35 x_{k}^{2} \\
& \quad-0.85 y_{k}^{2}+1.2 x_{k} y_{k}+0.17\end{aligned}$ & $\begin{array}{l}0.5862 \\
0.5862\end{array}$ & $\begin{array}{l}0.9984 \\
0.9984\end{array}$ & $\begin{array}{l}-1.44 \\
-0.23\end{array}$ & $\begin{array}{c}0.0388 \\
-0.0408\end{array}$ & 1.9505 \\
\hline
\end{tabular}

a computer search program was used to explore the strange hidden attractors, and some typical strange hidden attractors with no fixed point and with a single stable fixed point were presented in the phase-basin portrait. As can be observed from these phase-basin portraits, the basins of hidden attractors are very small in some cases, and therefore it is vital to investigate the hidden dynamics of these maps. In this paper, we only studied a special class of two-dimensional maps, but the proposed schematic method can be generalized to other maps. The future work would be to investigate the mechanism of hidden dynamics and the evolution of their basins of attraction in high-dimensional maps.

\section{Acknowledgements}

The authors are grateful to the anonymous reviewers for their valuable comments and suggestions that have helped to improve the presentation of the paper. This work is partially supported by the National Natural Science Foundation of China (Grant No. 11402224, 11202180, 61273106, 11171290, 11401543), the Natural Science Foundation of Jiangsu Province of China (Grant No. BK20151295), the Qin Lan Project of the Jiangsu Higher Education Institutions of China, the Jiangsu Overseas Research and Training Program for University Prominent Young and Middle-aged Teachers, the Fundamental Research Funds for the Central Universities, China University of Geosciences (Wuhan) (No. CUGL150419) and Presidents and the Top-notch Aca- 
demic Programs Project of Jiangsu Higher Education Institutions.

\section{References}

[1] Hénon, M.: A two-dimensional mapping with a strange attractor. Commun. Math. Phys. 50, 69-77 (1976)

[2] Leonov, G.A., Kuznetsov, N.V., Kuznetsova, O.A., Seledzhi, S.M., Vagaitsev, V.I.: Hidden oscillations in dynamical systems. Trans. Syst. Control 6, 54-67 (2011)

[3] Leonov, G.A., Kuznetsov, N.V.: Hidden attractors in dynamical systems: from hidden oscillation in HilbertCKolmogorov, Aizerman and Kalman problems to hidden chaotic attractor in Chua circuits. Int. J. Bifurcat. Chaos 23, 1330002 (2013)

[4] Bragin, V.O., Vagaitsev, V.I., Kuznetsov, N.V., Leonov, G.A.: Algorithms for finding hidden oscillations in nonlinear systems. The Aizerman and Kalman conjectures and Chuas circuits. J. Comp. Syst. Sci. Int. 50, 511-543 (2011)

[5] Leonov, G.A., Kuznetsov, N.V., Vagaitsev, V.I.: Localization of hidden Chuas attractors. Phys. Lett. A 375, 2230-2233 (2011)

[6] Leonov, G.A., Kuznetsov, N.V., Vagaitsev, V.I.: Hidden attractor in smooth Chua systems. Physica D 241, 1482-1486 (2012)

[7] Wei, Z.: Dynamical behaviors of chaotic systems with no equilibria. Phys. Lett. A 376, 102-108 (2011)

[8] Jafari, S., Sprott, J.C., Golpayegani, S.: Elementary chaotic flows with no equilibria. Phys. Lett. A 377, 699-702 (2013)

[9] Wei, Z., Wang, R., Liu, A.: A new finding of the existence of hyperchaotic attractors with no equilibria. Math. Comput. Simul. 100, 13-23 (2014)

[10] Molate, M., Jafari, S., Sprott, J.C., Golpayegani, S.: Simple chaotic flows with one stable equilibrium. Int. J. Bifurcat. Chaos 23, 1350188 (2013)

[11] Wei, Z., Zhang W.: Hidden hyperchaotic attractors in a modified Lorenz-Stenflo system with only one stable equilibrium. Int. J. Bifurcat. Chaos 24, 1450127 (2014) 
[12] Wei, Z., Yang, Q.: Dynamical analysis of a new autonomous 3-D system only with stable equilibria. Nonlinear Anal. Real World Appl. 12, 106-118 (2011)

[13] Jafari, S., Sprott, J.C.: Simple chaotic flows with a line equilibrium. Chaos Solitons Fractals 57, 79-84 (2013)

[14] Wang, X., Chen, G.R.: Constructing a chaotic system with any number of equilibria. Nonlinear Dyn. 71, 429-436 (2013)

[15] Chudzik, A., Perlikowski, P., Stefanski, A., Kapitaniak, T.: Multistability and rare attractors in van der Pol-Duffing oscillator. Int. J. Bifurcat. Chaos 21, 1907 C1912 (2011).

[16] Dudkowski, D., Prasad, A., Kapitaniak, T.: Perpetual points and hidden attractors in dynamical systems. Phys. Lett. A 379, 2591-2596 (2015)

[17] Prasad, A.: Existence of perpetual points in nonlinear dynamical systems and its applications. Int. J. Bifurcat. Chaos 25, 1530005 (2015)

[18] Sprott, J.C., Wang, X., Chen, G.R.: Coexistence of point, periodic and strange attractors. Int. J. Bifurcat. Chaos 23, 1350093 (2013)

[19] Li, C., Sprott, J.C.: Coexisting hidden attractors in a 4-D simplified Lorenz system. Int. J. Bifurcat. Chaos 24, 1450034 (2014)

[20] Leonov, G.A., Kuznetsov, N.V., Kiseleva, M.A., Solovyeva, E.P., Zaretskiy, A.M.: Hidden oscillations in mathematical model of drilling system actuated by induction motor with a wound rotor. Nonlinear Dyn. 77, 277-288 (2014)

[21] Lü, J.H., Chen, G.R.: Generating multiscroll chaotic attractors: theories, methods and applications. Int. J. Bifurcat. Chaos 16, 775 C858 (2006)

[22] Liu, C.X., Yi, J., Xi, X.C., et al.: Research on the multi-scroll chaos generation based on Jerk Mode. Procedia Eng. 29, 957C961 (2012)

[23] Ma, J., Wu, X.J., Chu, R.T., Zhang, L.P.: Selection of multi-scroll attractors in Jerk circuits and their verification using Pspice. Nonlinear Dyn. 76, 1951-1962 (2014)

[24] Jafari, S., Pham V.T., Kapitaniak T.: Multi-scroll chaotic sea obtained from a simple 3D system without equilibrium. Int. J. Bifurcat. Chaos 26, 1650031 (2016) 
[25] Sprott, J.C.: Strange attractors: creating patterns in chaos. M\&T books, New York (2000)

[26] Elhadj, Z., Sprott J.C.: 2-D quadratic maps and 3-D ODE systems: a rigorous approach. World Scientific, Singapore (2010)

[27] Luo, A.C.J.: Discrete and switching dynamical systems. Higher Education Press, Beijing (2012)

[28] Medio, A., Lines, M.: Nonlinear dynamics a primer. Cambridge University Press, Cambridge (2002)

[29] Heatha, W.P., Carrasco, J., Senb M.: Second-order counterexamples to the discrete-time Kalman conjecture. Automatica 60, 140-144 (2015)

[30] Zhusubaliyev, Z.T., Mosekilde, E.: Multistability and hidden attractors in a multilevel DC/DC converter. Math. Comput. Simulat. 109, 32-45 (2015)

[31] Jafari, S., Pham, T., Moghtadaei, M., Kingni, S.T.: The relationship between chaotic maps and some chaotic systems with hidden attractors. Int. J. Bifurcat. Chaos, accepted (2016)

[32] Sprott, J.C.: Elegant chaos: algebraically simple chaotic flows. World Scientific, Singapore (2010)

[33] Kuznetsov, N.V., Leonov, G.A.: A short survey on Lyapunov dimension for finite dimensional dynamical systems in Euclidean space. http://arxiv.org/pdf/1510.03835v2.pdf (2015)

[34] Leonov, G.A., Kuznetsov, N.V., Mokaev, T.N.: Homoclinic orbits, and self-excited and hidden attractors in a Lorenz-like system describing convective fluid motion. Eur. Phys. J. Special Topics 224, 1421-1458 (2015)

[35] Kuznetsov, N.V., Mokaev, T.N., Vasilev, P.A.: Numerical justification of Leonov conjecture on Lyapunov dimension of Rossler attractor. Commun. Nonlinear Sci. Numer. Simulat. 19, 1027-1034 (2014)

[36] Kuznetsov, N.V., Alexeeva, T.A., Leonov G.A.: Invariance of Lyapunov exponents and Lyapunov dimension for regular and irregular linearizations. Nonlinear Dyn., doi: 10.1007/s11071-016-2678-4 (2016) 
[37] Leonov, G.A., Kuznetsov, N.V.: Time-varying linearization and the Perron effects. Int. J. Bifurcat. Chaos 17, 1079-1107 (2007)

[38] Kuznetsov, N.V., Leonov, G.A.: On stability by the first approximation for discrete systems. 2005 International Conference on Physics and Control (PhysCon 2005), vol. Proceedings Volume 2005, IEEE, art. num. 1514053, pp. 596-599 (2015) 